\title{
Un primo sondaggio sulla sintassi dell'ultimo Pizzuto: considerazioni (e divagazioni) su Ultime e Penultime
}

\author{
Marco Carmello \\ Universidad Complutense de Madrid \\ carma.74@libero.it
}

\begin{abstract}
In quest'articolo si analizza la fase finale della produzione letteraria di Antonio Pizzuto, prendendo in considerazione Ultime e Penultime.

Tenendo conto del particolare sperimentalismo linguistico di quest'autore, ci si concentra sulla struttura linguistica analizzando, da un punto di vista sintattico, pragmatico e retorico la sua produzione, proponendo così un'interpretazione testuale che intende spiegare le ragioni poetologiche dell'opera pizzutiana.

L'articolo si chiude con un'analisi microtestuale di Dall'Ombra (dall'opera Ultime e Penultime) a riprova della ricerca proposta.
\end{abstract}

Parole chiave: Antonio Pizzuto; sintassi; stile; stilistica; pragmatica; predicato; rema.

\section{Abstract}

The article analyzes the last stage of Antonio Pizzuto writing activity with a particular reference to Ultime e Penultime.

Starting from the Pizzuto's linguistic sperimentalism, the article aims to analyze the authorial production from a sytactic, pragmatic and rhetoric point of view and reaches to a textual interpretation of the poetic reasons that inspire the Pizzuto's writing.

Finally, a micro-textual analysis of Dall'Ombra (from Ultime e Penultime) gives some proofs in accordance with our research.

Keywords: Antonio Pizzuto; syntax; style; stylistic; pragmatic; predicate; rhema. 


\section{Alcune considerazioni preliminari}

Dedicheremo questo lavoro ad uno degli autori più affascinanti del novecento letterario italiano: Antonio Pizzuto. Sarà bene stabilire subito che il nostro non sarà un lavoro di critica letteraria strictu sensu; noi affronteremo l'enigma Pizzuto da un punto di vista non nuovo ma, riteniamo, mai approfonditamente indagato: quello squisitamente linguistico o, per meglio dire, sintattico.

Già dai primordi della fortuna critica di Pizzuto - forse scarsa, ma gloriosa, non solo per i nomi dei primi esegeti e scopritori, ma soprattutto per l'acribia esemplare dei cultori che all'opera del Siciliano si sono, in questo torno di millennio, dedicati- praticamente tutti hanno messo l'accento sulla singolarissima forma linguistica degli scritti pizzutiani, forma che si è, nelle ultime prove, ${ }^{1}$ andata sempre più facendo rastremata ed estrema.

Contini, che se di Pizzuto non fu il primissimo estimatore ${ }^{2}$ certo fu il primo e più intimo critico, ha il merito di aver indicato la strada linguistica, seppur con un' «ipotesi cinese» ${ }^{3}$ che non ci sentiamo del tutto di condividere.

Pizzuto stesso è ben conscio della sua propria particolarità linguistica, come dimostrano interventi quali Sintassi nominale e pagelle, Paragrafi sul raccontare oltre ai giudizi sparsi nel corso dell'intervista con Paola Peretti apparsa postuma col titolo Pizzuto parla di Pizzuto, ${ }^{4}$ che è cifra precipua ed identificativa della sua ricerca estetica — sarebbe restrizione invalidante una precisa comprensione dell'opera pizzutiana parlare di semplice ricerca stilistica- e quindi perseguita sulla base di una ben precisa posizione filosofica e di un'attenta scelta di letture. ${ }^{5}$

1. Non solo quindi in Ultime e penultime, qui analizzate, ma anche in Pagelle I e II, Testamento, Giunte e virgole e Spegnere le caldaie, insomma nella produzione successiva a Paginette, individuato, già da Pizzuto medesimo, come giro di boa del suo percorso artistico.

2. Toccando ad altri la priorità temporale, in primis ad editori «impegnati» come Roberto Lerici, che di Pizzuto editò quasi tutti i lavori fino al passaggio al Saggiatore di Alberto Mondadori, e Vanni Scheiwiller, che di Pizzuto fu attento amico ed acuto corrispondente: proprio Scheiwiller favorì l'incontro fra l'autore siciliano ed il filologo di Domodossola.

3. Cfr. Gianfranco Contini "Guida breve a Paginette», in ID., Varianti e altra linguistica, Torino: Einaudi, 1970, p. 621-625.

4. Cfr. Antonio Pizzuto «Paragrafi sul raccontare», in ID., Paginette, Firenze: Polistampa, 2002, p. 119-120; ID. «Dello scrivere difficile», Nuovi Argomenti, n. 14, 1969, p. 55-64. Inoltre: Antonio Pizzuto / Paola Peretti Pizzuto parla di Pizzuto, con un'introduzione di W. Pedullà, Cosenza: Lerici, 1978. A riguardo sono importanti anche gli epistolari Contini (cfr. Antonio Pizzuto / Gianfranco Contini, Coup de foudre. Lettere (1963-1976), a cura di G. Alvino, Firenze: Polistampa, 2000), Nencioni (cfr. Antonio Pizzuto / Giovanni NenCioni, (1998) Caro Testatore, Carissimo Padrino. Lettere (1966-1976), a cura di G. Alvino, Firenze: Polistampa, 1998), Scheiwiller (cfr. Antonio Pizzuto / Vanni Scheiwiller, Le carte fatate. Carteggio (1969-1975), a cura di C. Gibellini, Libri Scheiwiller, 2005) e Spinelli (cfr. Antonio Pizzuto / Salvatore Spinelli, S. Ho scritto un libro ... Lettere (1929-1949), a cura di A. Pane, Palermo: Nuova Ipsa, 2001).

5. A riguardo si tengano presenti due fatti: l'influenza del pensiero del filosofo palermitano Cosmo Guastella e della sua personale teoria della conoscenza, basata su fenomenismo radicale e raffinato, su Pizzuto; in secondo luogo si consideri come Pizzuto riconosca in Platone uno degli autori che più di tutti hanno avuto influenza sulle sue scelte stilistiche 
Per i nostri scopi però le posizioni estetiche di Pizzuto, e la filosofia che le ispira, sono premessa, necessaria ma implicita, al punto di partenza della nostra analisi: la lingua di Pizzuto. Il motivo d'interesse di quest'oggetto è presto detto: la concezione che il Questore ebbe della narrazione, il suo fenomenismo rigoroso, il suo spontaneismo, lo portano ad agire direttamente sulla sintassi italiana eliminando le categorie del tempo, del modo e riducendo l'uso verbale alle solo forme nominali - infinito, participio e, più raramente, gerundiousate, come l'Autore in alcune note ad Ultime e penultime espressamente indi$\mathrm{ca}$, spesso in funzione non verbale.

Al silenziamento del verbo fa da contraltare non solo l'estensione della categoria nominale, ma la sua incipiente crisi: il nome, ed i gruppi nominali, non assumono solo funzioni narranti proprie del verbo - da qui l'ipotesi «cinese» del Contini-, ma il nome si impone sulla categoria aggettivale, altra parte del discorso che l'estetica pizzutiana radicalmente rigetta, ${ }^{6}$ annettendosela e lambisce addirittura le categorie funzionali della lingua, come le preposizioni (famoso resta l'uso del «nome preposizione», di cui proprio Ultime e penultime danno un bell'esempio).

Una lettura, anche rapida, per quanto possibile considerando l'oggetto, di Pizzuto, persino una lettura che si fermi al di qua di Paginette, permette di concludere che per il Questore siciliano il nome è l'unico cardine del narrare, o, altrimenti detto: lo spontaneismo fenomenista che impronta la teoresi e, conseguentemente, l'estetica pizzutiana, corrisponde linguisticamente al solo nome.

Se questo è l'esito finale della filosofia di Pizzuto, si pone un problema notevole: Pizzuto non può essere iscritto all'anagrafe dei grandi maccheronici, poiché la sua non è dissoluzione di linguaggi ma è propriamente dissoluzione linguistica. ${ }^{7}$ Pizzuto non può neppure essere annesso alle fila dell'avanguardia, ${ }^{8}$ poiché la sua non è contestazione semantico / semiotica al linguaggio ma è piuttosto contestazione gnoseologica; Pizzuto infine non può nemmeno essere liquidato come rarefatto calligrafo, poiché le sue "lasse» e "pagelle» hanno chiavi di perspicuità che escludono la bellezza del grafismo puro come ultimo fine. ${ }^{9}$ Eppure è innegabile che l'opera pizzutiana si assuma scientemente la taccia di agrammaticalità, facendone addirittura la sua cifra più intima e più esibita: si dovrebbe quindi concludere, per esprimerci in termini coseriani, che Pizzuto risalga oltre il sistema, che cioè abbandoni la struttura linguistica stessa, cosa, come è noto, impossibile per Coseriu, e non solo.

(e, si badi, che di magistero esclusivamente linguistico, e non filosofico, si tratta); l'altro è, non inaspettatamente, Joyce.

6. In accordo a quello spontaneismo fenomenico che impone di «narrare» e non di «raccontare», ossia «registrare», come appunto fa l'aggettivo (cfr., Pizzuto, Paragrafi ..., cit.).

7. In questa differenza, crediamo, si iscrive tutta la complessità del rapporto di Pizzuto con Gadda.

8. "Avanguardista a novant'anni», commentava, con sicula sagacità, Pizzuto stesso (cfr. PIzzuto / Peretti, Pizzuto parla di Pizzuto, cit., p. 56).

9. Ancora una volta si veda quel che il medesimo Pizzuto dice riguardo alla questione della forma e, sostanzialmente, dell'art pour l'art (cfr. Pizzuto / Peretti, Pizzuto parla di Pizzuto, cit., p. 8-56, in cui l'autore definisce anche i suoi presupposti filosofici). 
Ma sarebbe corretto concludere che Pizzuto, per ardire di troppa temerarietà, dissolva la sua ricerca linguistica nell'inanità dell'incomprensibile? ${ }^{10}$

Per rispondere a questa domanda bisogna che si scenda a sondare la sintassi pizzutiana.

\section{Abbozzo di un quadro teorico}

Qual è però l'attrezzatura migliore per tale speleologia? Ricordando che la nostra è un'analisi linguistica, e non letteraria, del linguaggio pizzutiano, dobbiamo chiederci quale approccio linguistico risulti più utile ad un corretto tratteggio dell'opera di questo autore.

In realtà la domanda che ci siamo posti prevede una scelta duplice: l'analisi va incentrata sul livello strutturale della lingua o su quello stilistico / pragmatico?

\subsection{Nibil est in sintaxi quod prius in stilo non fuerit?}

In Tombeau de Leo Spitzer ${ }^{11}$ Gianfranco Contini attribuisce proprio a Leo Spitzer il motto, più o meno scherzoso, che abbiamo scelto come titolo di questo paragrafo, solo che, nella versione originale, si tratta di un'affermazione, non di una domanda.

Comunque si voglia intendere il latino stilum — sia letto nel suo senso proprio di «stilo» (bastoncino appuntito con cui i Romani incidevano lettere sulle tavolette incerate), e quindi inteso come volontà dell'autore / parlante; sia invece interpretato nel suo significato metonimico di «stile», e quindi istituzione linguistica che più autori / parlanti condividono ${ }^{12}$ —, a far problema è il termine sintaxis ${ }^{13}$ come l'iperonimo "grammatica", anche sintassi ha, nella nostra cultura, due differenti significati, l'uno al di là, l'altro al di qua dello stilum comunque inteso.

Se per sintassi s'intende la normazione dei costrutti linguistici, allora se ne può concludere che il motto spitzeriano abbia possibilità, più o meno buone,

10. Ci pare questa, in buona sintesi, la posizione sostenuta da Cesare Segre (cfr. Cesare SEgre, "L'Hypnopaleoneomachia' di Pizzuto", in ID., I segni e la critica, Torino: Einaudi, 1969, p. 209-227), non a caso l'unico critico verso cui Pizzuto esprime allarme, più che fastidio (cfr. Pizzuto / Contini, Coup..., cit., lettere del 16 e del 19 luglio 1967, e Pizzuto / SCHeIwILler, Le carte..., lettera del 17 luglio 1967).

11. Varianti ed altra linguistica, cit., p. 650-660.

12. C'è da credere che per Spitzer la distinzione fosse sì cosa di non poco conto ma non così traumatica, potendo essere lo «stile» («collettivo») frutto dello «stilo» («personale») dei singoli; tuttavia, la distinzione che abbiamo accennato qui, ebbe, almeno ci sembra, importanti conseguenze nell'ambito della «stilistica». Per rimanere alla sola Italia (il discorso per la Francia si farebbe più complesso, basti solo notare che per Bailly sarebbe difficile parlare di stilum come «stilo», volontà dunque, invece che di stilum come «stile», quindi istituzione linguistica condivisa), la distinzione ha provocato una proficua tensione, più sul versante filologico, basti citare il solo nome del Contini, che su quello linguistico.

13. Di cui si dovrà sempre tener presente l'originale senso greco di «disposizione ordinata di più elementi». 
a seconda di come si vedano i fatti della lingua, di accampare quarti di verità: se però per sintassi s'intendono le regole di struttura che organizzano la lingua e ne permettono l'uso, allora è vero esattamente il contrario, ossia che: nibil est in stilo quod prius in sintaxi non fuerit.

Le due idee di sintassi, e in realtà dovremmo ormai dire le due idee di grammatica, che abbiamo sunteggiato sopra si scontrano entrambe con l'esperienza pizzutiana, risultando inutili ad una definizione della lingua del Siciliano.

Se cercassimo di intendere il nostro oggetto partendo dal primo dei due opposti presupposti (nihil est in sintaxi quod prius in stilo non fuerit), ossia analizzando Pizzuto dal punto di vista della pragmatica o da quello dei meccanismi di coesione / coerenza / connettezza ${ }^{14}$ testuale, si dovrebbe concludere che il testo di Pizzuto, dal punto di vista della narrazione, si sfrangia, in una serie di atomi autonomi, spesse volte limitati ad un unico nome, che vivono gli uni accostati agli altri chiusi in monadologica indifferenza gli uni verso gli altri, rimanendo l'unico filo dello scritto quello che gli dona la carità del lettore. E proprio tale carità respinge anche un'interpretazione via pragmatica.

Se infatti dovessimo applicarci al testo pizzutiano prendendo le mosse da una rigorosa linea austiniano-griceana avremmo una duplice possibilità: o intendere tutta l'opera di Pizzuto, o almeno quella dell'ultimo Pizzuto, come una sorta di ipertrofico atto linguistico che pone in essere la personale «narrazione» ${ }^{15}$ dell'autore; "narrazione» a cui il lettore attenderebbe come il fedele attende alla salmodia durante la messa. Dunque un autore-messaggio, con buona pace della poetica di questo stesso autore, che contro il "messaggio» dispone salve di finissima e dissolutrice condanna.

L'altra via pragmatica sarebbe quella di integrare al testo quello che nel testo mancherebbe, individuandone i suggerimenti reconditi. Il testo pizzutiano è però pensato in maniera tale che ogni integrazione ne distruggerebbe il senso, perciò i suggerimenti che in esso pure si scoprono restano appunto suggerimenti puri, ${ }^{16}$ ossia agenti fuori dal testo, senza però diventare indicazioni che permettano di completare le oscurità del senso. Altrimenti detto, il testo pizzutiano non ammette reinterpretazione. ${ }^{17}$

14. Per i concetti elencati sopra si vedano Heinrich Weinrich, Tempus. Besprochene und erzählte Welt, Stuttgart: Kolhammer, 1964; János Sandor PetőFI, Scrittura e interpretazione. Introduzione alla testologia semeiotica dei testi verbali, Roma: Carocci, 2004; MariaElisabeth Conte, Condizioni di coerenza. Ricerche di linguistica testuale, nuova edizione con l'aggiunta di due saggi a cura di Bice Mortara Garavelli, Alessandria: dell'Orso, 1999; Eugenio Coseriu, Linguistica del testo. Introduzione a una ermeneutica del senso, Roma: Carocci, 1997.

15. Uso fra virgolette i termini narrare, narrazione, raccontare, racconto ecc. quando mi riferisco alla concezione del narrare definita da Pizzuto medesimo nei Paragrafi sul raccontare (cfr. Pizzuto, Paragrafi..., cit.).

16. Non a caso il Questore siculo assimila fra loro prosa e poesia (cfr. Pizzuto / Peretti, Pizzuto parla di Pizzuto, cit., p. 61-64).

17. Uso reinterpretazione nel senso di M. E. Conte (cfr. Conte, Condizioni..., cit.): la reinterpretazione così intesa è dunque un concetto tecnico della pragmatica testuale che definisce quel particolare processo di comprensione del testo basato sulla possibilità di rifondere i 
Se, considerato il fallimento del precedente approccio, ci affidassimo invece all'altro presupposto di partenza (nihil est in stilo quod prius in sintaxi non fuerit), potremmo ottenere risultati più interessanti solo se riuscissimo a superare un imponente ostacolo di partenza: l'agrammaticalità patente di Pizzuto.

Rimanendo fermi a questo dato, o si conclude per la semplice incomprensibilità, o si adotta la strada, affatto montaliana, della parafrasi testuale, con relativo travisamento dell'opus pizzutiano.

Il problema posto dal secondo approccio non è però superabile internamente a quest'approccio, a meno che non si assuma la possibilità di poter individuare due differenti sistemi all'interno della grammatica: quello sintattico, al di qua dello stilum, e quello retorico, al di là dello stilum, reintegrando così l'approccio precedentemente esaminato e limitando non la forza ma l'autonomia esplicativa dei due differenti approcci.

Il problema che questa soluzione pone è però duplice: primo, cosa autorizza una moltiplicazione dei sistemi che concorrono a comporre la lingua; secondo, come si integrano i due sistemi?

\subsection{Sintassi e retorica}

In che modo la retorica può essere definita un «sistema»? E cosa si intende qui per «retorica»? La risposta alla seconda domanda implica non tanto la soluzione della prima - è ormai risaputo che la retorica sia un sistema ${ }^{18}$-, quanto il «tono» del quesito.

Possiamo opporre retorica a grammatica in quanto la prima riguarda il sistema dell'esporre, del pronunciare, del recitare, dello scrivere un preciso testo, mentre la seconda pertiene al dire, al performare, allo scrivere come atto generale. Non è perciò del tutto corretto assimilare l'opposizione grammatica I retorica alla ben nota distinzione saussuriana langue / parole, poiché, se la retorica non tange la langue limitandosi ad un ambito specifico della parole, la "grammatica» non è limitata alla sola langue, essa tange anche alcuni non irrilevanti fenomeni di parole.

La sovraestensione del concetto di "grammatica» che la nostra esposizione comporta ha un effetto anche sull'uso del concetto di «retorica» qui proposto: adottando lo schema espositivo del Lausberg, ${ }^{19}$ possiamo dire che, fra le figu-

diversi elementi costitutivi del testo stesso sulla base di una nuova informazione presentata dal racconto. Tipicamente è il procedimento proprio della letteratura poliziesca, in cui le informazioni contenute nel finale permettono una reinterpretazione di tutti gli aspetti più rilevanti della trama. In termini pizzutiani è questo un procedimento del «raccontare», quindi a priori escluso.

18. A riguardo si vedano il classico manuale del Lausberg (Heinrich LAUsBerg, Elemente der literarischen Retorik, München: Max Hueber Verlag, 1967) e la «storia» della retorica di Vickers (Brian Vickers, In Defence of Rhetoric, Oxford: Clarendon Press, 1998).

19. Il manuale del Lausberg è ormai classico; trovandosi però, nell'evoluzione dell'attuale lessico scientifico-saggistico, l'aggettivo "classico» ad aver ormai assunto accezione di vox media significante, pressapoco, "studio che riassume egregiamente e con invidiabile ricchezza informativa una linea di studi, ma ormai superato» (giusta la potenza del principio 
re dell'ornato in verbis singulis, i tropi di spostamento e di limite, fra le figure dell'ornato in verbis coniunctis, le figure per ordinem, sia nell'ambito delle figurae elocutionis sia in quello delle figurae sententiae, l'immutatio sintactica ed ancora una volta i tropi di spostamento e di limite nel solo ambito delle figurae sententiae, appartengono, secondo la nostra esposizione, alla "grammatica" non alla «retorica». ${ }^{20}$

La «retorica» viene così ad avere un ambito doppio: sua è tutta l'ampia regione della topica, quindi la teoria dell'occasione come criterio della scelta di argomento, stile, colore adeguato all'occasione linguistica; sua è anche quella regione di ricerca di non usuali nessi semantici, di non comuni occasioni d'uso, di segnalazioni linguistiche che definiscono e creano l'occasione retorica, dunque della «retorica» in questo senso sono la metafora, la metonimia, l'ironia, l'amplificatio, la ripetizione, il salto logico nella connessione argomentale. ${ }^{21}$ Questa seconda regione della «retorica» si incontra con quella parte di

ispiratore della più moderna ricerca, per cui recentiora meliora sed novissima optima), bisogna giustificare il costante riferirsi al manuale del Lausberg per una descrizione del sistema retorico, elidendo così tutta quella rinascita degli studi di retorica, iniziata col lavoro di Perelman e Olbrechts-Tyteca (Chaim Perelman / Lucie Olbrechts Tyteca, Traité de l'argumentation. La nouvelle rhétorique, Paris: PUF, 1958). Ebbene, la scelta è dovuta precisamente all'intento di elidere la «neo-retorica» dall'orizzonte di questo scritto, non certo per una qualche forma di sciovinismo, ma perché l'intento di fare della retorica il fondamento di una generale scienza del linguaggio, che ci pare unanimemente perseguito, pur nella evidente disparità dei metodi, dalle «neo-retoriche», non riguarda il nostro tentativo di usare l'opposizione grammatica / retorica come lente di rifrazione di alcuni fenomeni linguistico-letterari.

20. Questi i riferimenti all'edizione italiana del manuale del Lausberg (LAusberg, Elemente..., cit.): tropi di spostamento e di limite in verbis singulis, p. 107-122, uguali ai $₫ 184-215$; figure in verbis coniunctis: figure per ordinem nelle figurare dell'elocuzione, p. 180-194, uguali ai $\$ 329-362$, figure per ordinem nelle figurare sententiae, p. 229-230, uguali ai $\$$ 212-215, immutatio sintactica, p. 245-246, uguali ai $\$ 244-247$, tropi di spostamento e di limite p. 231-234, uguali ai $\$ 418-421$.

21. Può parere strano che alcuni dei fenomeni elencati siano iscritti alla parole, soprattutto pare strano che tale iscrizione riguardi metafora e metonimia, che invece, particolarmente in approcci lessicologici di tipo cognitivista o generativista, sono visti come fenomeni di sistema. Sebbene non sia questa la sede per affrontare il complesso fenomeno della metafora, noi assumiamo che la ricerca di una «struttura della metafora» sia destinata ad un fallimento: adottando una posizione a là Gross (Gaston Gross, Les expressions figees en francaise: noms composes et autres locutions, Paris: Gap, Paris, 1996), si potrebbe dire che non esista un vero e proprio meccanismo metaforico / (metonimico), esistono piuttosto collocazioni lessicali non contigue. Considerando che il parlante ha, nella scelta della varietà lessicale — varietà intesa sia come sinonimia sia come variatio nella scelta di accostamento- ampia libertà, possiamo concludere che la metafora scatti come interpretazione suppletiva laddove un accostamento lessicale non contiguo renda opaca l'interpretazione semantica, significativamente però la metafora non ha interpretazioni univoche, neppure laddove essa obbedisca, come nel caso della catacresi, ad esigenze di dominazione che ne limitano pesantemente la polisemia. Ad esempio, il semplice enunciato: «i meccanismi di censura sessuale erano in epoca vittoriana tanto forti da indurre a coprire le gambe dei tavoli con lunghe tovaglie», non sarebbe del tutto perspicuo se non si potesse riportare la catacresi «gambe dei tavoli» sarebbe meglio dire il nome catacretico, non essendovi altro nome in italiano per questa parte del tavolo- alla sua origine metaforica, per cui è possibile il passaggio semantico gambe del 
«grammatica» cui, poco sopra, abbiamo attribuito giurisdizione su alcuni mezzi della retorica classica.

Il concetto classico di retorica, che noi avevamo diminuito per mezzo dell'opposizione con una cosiddetta "grammatica», viene così a ricomporsi, ma è una ricomposizione che svela come l'ambito retorico, riassunto nella sua versione "classica» e "canonica» dal Lausberg, sia, se analizzato con gli occhi della linguistica contemporanea, un campo di incontro di differenti fatti linguistici, solo alcuni dei quali possono essere ricondotti all'asistematicità dell'istituzione letteraria o dello «stile».

Del resto, la ricomposizione del concetto classico di retorica così ottenuta dissolve anche l'altro polo dell'opposizione, ossia la «grammatica», che può essere suddivisa in due grandi regioni: i fatti strutturali della lingua, quella competenza, e parliamo ormai in termini generativisti, che caratterizza l'uso di ogni parlante, ed i fatti della codificazione, ${ }^{22}$ ossia quell'insieme di fatti linguistici che sono individuati come oggetto del discorso normativo attorno ad una lingua. ${ }^{23}$

Notiamo di sfuggita come anche nella seconda accezione di "grammatica» non sia sempre vero che nibil est in sintaxi quod prius in stilo non fuerit per almeno due buone ragioni: la prima, il processo normativo, anche quando, come è il caso dell'italiano, del latino o, mutatis mutandis, del francese, sia estremamente pervasivo, appare sempre come un processo selettivo che opera sulla lingua di uno o più autori sotto la guida di ragioni linguistiche extrastilistiche; ${ }^{24}$ la secon-

tavolo $\rightarrow$ gambe umane $\rightarrow$ eccitazione sessuale. L'enunciato esempio, sebbene non sia sintatticamente o semanticamente ambiguo, basa parte del suo significato composizionale sulla possibilità di ricostruzione di una catena metaforica: si potrebbe obbiettare che l'esempio di polisemia catacretica fatto sopra non differisce molto dalla semplice polisemia nominale. In realtà non è così: si immagini che un ipotetico interlocutore ribatta all'enunciato esempio col seguente enunciato: «eccitarsi per le gambe del tavolo: erano ben perversi, questi vittoriani». Anche in questo caso la polisemia metaforica gioca, ma il legame è di segno inverso a quello definito sopra, qui infatti la catena è: gambe del tavolo $\rightarrow$ inanimato $\rightarrow$ perversione. Si potrebbero poi dare giochi metaforici complessi: ad esempio quello proposto da uno slogan pubblicitario di una mostra d'antiquariato, nella cui locandina appare la fotografia di un tavolo rococò sotto cui campeggia la frase: «ogni intenditore si ecciterà per queste gambe», dove è istituito un gioco di rimandi incrociati fra animato $\rightarrow$ inanimato / gusto $\rightarrow$ eccitazione. Come si vede dunque la varianza semantica della catacresi implica un'arbitrarietà interpretativa incomparabile rispetto a quella del nome, anche se evidentemente minore di quella della metafora pura, che non abbia subito un processo di «lessicalizzazione».

22. E questa un'etichetta che può parere inusuale, soprattutto perché la grammatica tutta è, o per lo meno può esser vista, come «codificazione»: nel prosieguo del discorso risulterà chiaro quali sono le ragioni che ci hanno spinto a preferire questa all'altra possibile definizione di "grammatica della norma».

23. "Norma» è nozione che non ha goduto, ed in parte ancora non gode, di buona stampa in ambito linguistico. Se la polemica contro la «buona norma» ebbe ragion d'essere in tempi nei quali veniva affermandosi la necessaria autonomia dell'oggetto lingua come campo neutro di indagine, oggi il problema della norma linguistica può essere recuperato, non solo in ambito storico-linguistico, $\mathrm{ma}$ anche in ambito prettamente linguistico, come punto critico della definizione di lingua.

24. Non a caso abbiamo citate le tre lingue di cultura europee che più di tutte sono state sottoposte ad un processo di normazione rigorosa. Eppure questa normazione è opera di una 
$\mathrm{da}$, sebbene la normazione risponda in apparenza ad esigenze «stilistiche» —quale lingua è giusto scrivere? quale l'espressione più elegante? quale il buon gusto? - in realtà essa nasce per soddisfare un' esigenza di correttezza che è anzitutto rivolta alle strutture della lingua e vuole anzitutto definire i "buchi» che per loro dialettica interna queste strutture non soddisfano - passato prossimo o remoto? a o per? uso di articolo o assenza di articolo?-, perciò ancora una volta la normazione nasce ed agisce indipendentemente dallo «stile».

Tirando le fila possiamo ora rispondere alle due domande di partenza: per retorica intendiamo quel particolare uso linguistico che intende creare significato a partire dalle discromie strutturali della lingua per mezzo dell'uso forzoso di strutture sintattiche e lessicali; in questo senso la retorica costituisce un sistema di regolarità (non di regole) che definiscono diversi tipi di scarto rispetto alla struttura linguistica di partenza determinandone il grado di distanza e definendone l'ambito.

Se per retorica si intende ciò, allora non è difficile vedere come la sintassi rappresenti il limite della retorica: ${ }^{25}$ abbiamo testé parlato di uso forzoso delle strutture sintattiche e lessicali, in realtà si potrebbe ridurre l'endiadi alla sola sintassi, poiché l'uso del lessico risulta forzoso solo in relazione alla sua collocazione all'interno della struttura sintattica, quindi solo in relazione alle codifiche sintattiche che il senso lessicale comporta, perciò in conclusione l'uso forzoso del lessico equivale ad un uso forzoso della sintassi tout-court.

Non discuteremo qui il problema della sintassi, né ci addentreremo nell'intricata questione della rappresentazione del sistema sintattico e degli approcci più adatti a tale descrizione: basti dire, nella più neutra delle maniere, che la sintassi rappresenta quel sistema che istituisce la regolarità e, conseguentemente, la condivisibilità e l'apprendibilità della lingua.

Stabilito così il rapporto fra retorica e sintassi, risulta evidente che l'opera letteraria, il lavoro dell'autore elidono l'asse sintattico, dato come condizione sottesa alle possibilità di scrittura, e si rivolgono all'asse retorico, che costituisce l'opera letteraria intesa come risultato dello scarto fra struttura e diffrazioni dovute alla creatività del singolo autore.

selezione: a far da modello non è la lingua di Cicerone, bensì la lingua depurata delle Verrine e delle Catilinarie, depurata dalle "scorie volgari» delle lettere o dall'eccessivo asianesimo di certi passaggi delle Filippiche o, ancora, dai tecnicismi delle opere teorico / filosofiche. $\mathrm{Nel}$ caso di una lingua in cui non sia individuato un autore preminente su cui condurre la normazione, si individueranno caratteristiche che daranno un'idea di style al cui vaglio passeranno i singoli: Racine apparirà così migliore di Corneille e Voltaire preferibile a Rosseau; fra i pansée pascaliani si indicheranno quelli che più di altri obbediscono ad una sorta di rochefoucauldiana témperance. È interessante notare come tutti questi canones possano essere smontati dall'interno, come già, per il canone italiano, faceva nel seicento in un suo libello Daniello Bartoli (Daniello BARTOLI, Il torto e l diritto del non si può dato in giudicio sopra molte regole della lingua italiana, esaminato da Ferrante Longobardi, cioè da P. D. B., Parma: Fondazione Pietro Bembo, Ugo Guanda Editore, 2009 [1655 $\left.{ }^{1}\right]$ ).

25. In questo senso è corretta l'idea di Coseriu secondo cui non si può risalire oltre il limite del sistema. 
Non analizzeremo minutamente questo scarto; ci sarà sufficiente dire solo questo, l'opera letteraria arriva a lambire, interrogare, agire sulle strutture sintattiche solo quando abbia raggiunto un alto grado di retorizzazione; quando cioè l'opera occupa uno spazio retorico vasto, quando tutti i meccanismi retorici non appartenenti alla grammatica, per riferirci all'opposizione su menzionata, siano stati impiegati, quando anche l'intera gamma dei meccanismi retorico-grammaticali è stata impiegata, allora è possibile che la volontà autoriale si sposti alle strutture della sintassi.

Però, questa discesa verso la sintassi è per solito bloccata dal mantenersi di uno spazio di separatezza fra ambito retorico ed ambito grammaticale, per cui la regolarità sintattica è sollecitata solo negli ambiti ristretti entro i quali tutto l'armamentario dei mezzi retorici è già stato esperito ed esaurito.

Se però esistono ragioni di poetica che sollecitano l'autore ad una ricerca linguistica tale per cui lo spazio retorico non è più sufficiente, allora si ha un'eversione assoluta del sistema retorico ed un passaggio dalla creatività linguistica direttamente al sistema sintattico: è questo il caso di Antonio Pizzuto. ${ }^{26}$

\section{Pizzuto eversore assoluto del sistema retorico: ovvero sulla direzione della sintassi pizzutiana.}

Il radicalismo della poetica pizzutiana spinge l'autore ad elidere l'ambito retorico, che, nella misura in cui è inevitabilmente legato alle ragioni sistematiche della sintassi, risulta insoddisfacente per le esigenze del fenomenismo di questa poetica. L'elisione dell'ambito retorico avviene tramite una saturazione vieppiù esaustiva dello spazio definito dal sistema retorico, saturazione che è facile delineare seguendo la linea evolutiva dell'autore: la prima fase produttiva, ancora ancillare rispetto al Pizzuto pubblico, ufficiale di polizia di alto livello, rappresenta più che altro l'acquisizione dei mezzi classici della scrittura; potremmo quasi dire che in questa prima fase, poi necessariamente rifiutata, alla luce della poetica pizzutiana che va facendosi, scritto dopo scritto, sempre più "opera», fino all'annullamento della classica distinzione poetica (teoria) / scritti (pratica), che risulta tanto spesso insanabile negli autori della generazione di Pizzuto, ${ }^{27}$ rappresenti l'allenamento del Questore nell'ambito del «racconto», di cui Pizzuto si impossessa forse già con fine eversivo.

La seconda fase, che si apre con quello che l'autore e, in accordo con lui, la critica considerano il vero lavoro di esordio, Signorina Rosina, e che comprende i lavori fino a Paginette, ossia: Si riparano bambole, Sul ponte di Avi-

26. Caso a cui, in tutta la storia della letteratura italiana, ritengo possa essere accostato, pur con le dovute cautele, un unico nome, mai peraltro citato da Pizzuto (e, a quanto ci consta, neppure dalla critica), quello di Teofilo Folengo.

27. Nella sua introduzione a Paginette (Antonio Pizzuto Paginette, Milano: Lerici, 1964, ora in Contini, Varianti..., cit., p. 621-625) il Contini nota come le date di Pizzuto (1893-1976) si facciano beffe di quella periodizzazione in "generazioni» — di cui si beffava l'autore stessoche tanta parte ebbe (ed ha) nella storiografia del novecento letterario italiano: il contemporaneo dei Cecchi e dei Bacchelli era sodale dei Sanguineti, ma su posizioni più avanzate! 
gnone, Ravenna, rappresenta il rifiuto del «raccontare» a favore del «narrare»; è questa la fase in cui Pizzuto affronta l'insufficienza del sistema retorico. Non è azzardato concludere che in questa seconda fase Pizzuto sperimenta l'impossibilità di essere "maccheronico»: non è per lui sufficiente la tradizione del mistilinguismo, della mistura di registri e livelli, dello scarto rapido, segnato dall'iperbato, dall'anacoluto, dallo spezzarsi continuo del ductus. Pizzuto non può quindi essere maccheronico nel senso in cui lo è un Gadda, ma neppure in quello, prettamente linguistico, in cui lo è, ad esempio, Folengo, che pure, fra gli autori del patrimonio italiano gli è forse il più vicino.

A differenza di Folengo però Pizzuto interroga il linguaggio radicalmente: mentre il materiale linguistico su cui operava l'invenzione di Folengo era lingua di scuola sottoposta alla tensione barbarizzante del sermo humilis et vulgaris, la cui grossezza irrompeva nelle ordinate campate del latino classico scompaginandole e ricomponendole secondo un ordine frutto dell'abile contraffazione di quello sconvolto, il materiale linguistico di Pizzuto è la sua stessa lingua materna. La scelta della lingua materna come oggetto di «maccheronee» porta Pizzuto in campo aperto: laddove Folengo si muoveva nei limiti ben fissati dell'elegante laboratorio rappresentato dal latino umanistico, facendo della sua scompaginazione una sorta di giardino di Adone in cui saggiare i limiti dell'espressività linguistica, Pizzuto esce dai limiti di un laboratorio qualsiasi e si imbarca direttamente nella selva del linguaggio. Non a caso, pur avendo in comune l'abilità neologistica, i due autori trovano in campi diversi della lingua le ragioni del loro sperimentare: Folengo si rivolge alla morfologia, ${ }^{28}$ laddove Pizzuto si imbarca, quasi a peso morto, nella sintassi.

Ed eccoci così giunti all'ultimo Pizzuto, quello di: Paginette, Pagelle I e II, Testamento, Ultime e penultime, Giunte e virgole, Spegnere le caldaie, in cui lo sperimentalismo linguistico assume potenza che si dovrebbe giustamente definire heideggeriana.

Stabilita l'impossibilità di «narrare» entro l'ambito retorico, che viene abbandonato al solo «racconto", la ricerca scende al livello sintattico e subito si volge alla creazione di una struttura linguistica fermamente antirematica, una sorta di lingua adamitica nella quale tutto diviene sforzo di dominazione, di ostensione, al di fuori ed al di là di ogni giudizio, ivi compresi quelli di tempo, modalità, agente, inerenti al verbo. ${ }^{29}$

A differenza però di quanto accade nell'ambito prettamente retorico, nell'ambito sintattico la ricerca ha un limite preciso, quello tracciato dalla comprensibilità: si possono «sabotare» alcune strutture, si possono evitare alcune classi di parole, si possono limitare o modificare le connessioni, ma non si può travalicare una soglia oltre la quale possono sì darsi elementi linguistici ma non linguaggio. ${ }^{30}$

28. Lo sperimentalismo sintattico del Folengo, che pure è rilevantissimo, non esce dall'ambito di ciò che qui abbiamo chiamato «retorica».

29. Il rema appunto nella tradizione grammaticale greca.

30. È solitamente questa soglia che viene superata in determinate patologie fisiche - i vari tipi di afasia, ad esempio- o psichiche — esemplari alcune forme di delirio paranoideo e 
Pizzuto, con la sua sperimentazione, arriva a quella soglia ma non la varca: è quindi giunto il tempo di fissare alcune caratteristiche generali della famosa «sintassi nominale» e quindi di analizzarne alcuni esempi.

\subsection{Sintassi notazionale ed antirematica}

La grande scoperta pizzutiana è la non necessità del verbo per ciò che egli definisce «narrazione»: ma cos'è la «narrazione» e come, ed a quale prezzo, è possibile realizzare in essa l'eliminazione del verbo, ossia del rema e delle connessioni logico-argomentative che esso definisce?

La contropartita necessaria dell'eliminazione del verbo è certamente l'esplosione della categoria nominale: in realtà però non basta istituire l'equazione meccanicistica eliminazione del verbo = potenziamento del nome per spiegare le cose; ${ }^{31}$ potenziamento del nome vuol infatti anche dire, e non è paradosso, crisi della categoria morfo-sintattica «nome».

Nella velata polemica con Gadda, che sembra percorrere buona parte dell'opera tardo pizzutiana, ${ }^{32}$ il Siciliano pare aver risolto uno dei problemi che più perseguitano il Lombardo, quello dell'opposizione io / tu, io / altro: ${ }^{33}$ l'abolizione del rema infatti comporta anzitutto l'abolizione delle categorie di agente / paziente, di attante / aggiunto, di ruolo tematico che sono definite in ambito sintattico dall'occorrere del verbo all'interno della struttura frasale.

Il grande problema del distacco fra soggetto scrivente ed altri, quindi implicitamente fra soggetto scrivente e linguaggio stesso, che Gadda poneva in termini patentemente pronominali (si veda il passo della Cognizione citato in nota), viene da Pizzuto riportato al piano sintattico: attraverso l'abolizione della gerarchia dei ruoli sintattici connessa all'elisione del rema, viene meno la struttura analitica dell'enunciato, ${ }^{34}$ non è quindi più possibile chiedersi cosa accada, non essendo possibile individuare agenti, pazienti, beneficiari, cause, fini di un'azione.

Il venir meno della notazione di tempo, l'effetto più patente della prosa pizzutiana ed anche il meglio evidenziato dall'autoanalisi dell'autore, altro non è, in realtà, se non una conseguenza dell'analiticità che la formula azionale del verbo, algebricamente descritta dai ruoli semantici insiti nella sua sintassi,

schizofrenico- (Janusz Wròbel Language and Schizophrenia, Philadelphia: Benjamins, 1990; Sergio Piro Parole di follia, Milano: Franco Angeli, 1992).

31. En passant noteremo come Pizzuto, sia nei Paragrafi sul raccontare (Pizzuto, Paragrafi..., cit.) sia in Sintassi nominale e Pagelle (Pizzuto, Sintassi..., cit.) favorisca quest interpretazione; è difficile dire se ciò avvenga per voluto intento dissimulatorio, per volontà di meglio risaltare il punto che Pizzuto riteneva veramente apicale della sua ricerca stilistica, o perché parte degli strumenti usati risultano effettivamente opachi all'autore medesimo.

32. Come meglio vedremo nell'analisi della prima «lassa» di Ultime: Dall'ombra (Antonio Pizzuto, Ultime e Penultime, edizione critica di Gualberto Alvino, Napoli: Cronopio, 2001).

33. Cfr. Carlo Emilio Gadda, La cognizione del dolore in ID., Opere di C. E. Gadda-Vol. I, Romanzi e racconti I, a cura di Dante Isella, Milano: Garzanti, p. 637-639.

34. Banalmente noteremo che un'analisi «logica» o del "periodo» su una pagina dell'ultimo Pizzuto è semplicemente impossibile. 
comporta. Venendo meno l'azione, viene meno il complesso campo in cui l'azione si dispiega, vale a dire il tempo.

Certamente il venire meno del tempo, nella sua doppia accezione di time e tense, permette il recupero dei nomina actionis, ossia quegli infiniti e quei participi che Pizzuto sente, in più occasioni, di dover indicare come privi di valore verbale, come «nomi» appunto. Tuttavia, anche quando l'infinito non sia abbassato al suo mero valore di nome, esso non assume mai una piena funzione verbale, contribuendo piuttosto a creare un nome complesso, una nota, il cui semplice aggancio con un agente o paziente forma un atomo, appena un poco più pesante, contrapposto agli altri atomi nominali che definiscono il coagulo della narrazione.

Più interessante ${ }^{35}$ invece l'uso del participio, che assume ruolo precipuamente aggettivale, quasi l'azione medesima non fosse altro che l'attributo accidentale di un nome: l'aggettivo è un altro dei punti critici della prosa pizzutiana, essendo elemento di delimitazione. È proprio nel non immediatamente percepibile distacco fra l'uso pizzutiano e quello comune dell'aggettivo che si percepisce soprattutto il tono della "narrativa» ${ }^{36}$ del nostro.

Nessi come: «Baritoni avanzi quantici distillativi in irrefrenabile stupendo sfacelo...», ${ }^{37}$ svelano come le strutture aggettivali, semplici, o complesse, via coordinazione participiale («distillativi in irrefrenabile stupendo sfacelo») siano connessi non per contrapposizione, secondo una strategia "cinese», come vorrebbe Contini, ma per inclusione ordinata, fino a formare un nome complesso, in cui il materiale linguistico si raccoglie quasi come in un composto denominale della lingua greca. L'ordine aggettivale istituisce il senso rotazionale del «nome»: anzitutto è in rilievo la profondità degli «avanzi» («baritoni»), che sono "quantici» in funzione della loro pertinenza alla struttura profonda della materia, e come tali si distillano («distillantivi») in quell'irrefrenabilità particolare definita dallo stupore («irrefrenabile stupendo») che, a sua volta, definisce lo «sfacelo».

Un rapporto dunque osmotico fra nome ed aggettivo, ma lo stesso rapporto osmotico che esiste fra nome e nome, lo stesso rapporto che consente l'uso non nominale del nome, come nel ben noto caso del nome "preposizione» («bassorilievo parete» per calorifero).

A questo punto è ormai chiaro che il carattere precipuo che Pizzuto cerca nella sintassi è la pura notazionalità: laddove il rema, nel suo significato primo etimologico di formula, atto di parola stabilito, parola d'ordine, ${ }^{38}$ istituisce un ordine che è già anche giudizio, rappresentazione, «racconto", insito nella natura stessa della forma linguistica, l'onoma, la cui etimologia

35. E, verrebbe da dire, più ironico.

36. «Narrativa» intesa come arte del «narrare» opposto al «raccontare», secondo l'ormai nota dicotomia «narrazione» / «racconto».

37. È l'incipit della lassa XXIX di Ultime: Elettronica, (Pizzuto, Ultime..., cit.).

38. Cfr. Pierre Chantraine Dictionaire étymologique de la langue grecque, Paris: Librairie Klincksieck, Paris, 1968-1980. 
si può connettere a quella del latino nota ${ }^{39}$ quindi segno, indizio, tacca, ma anche notazione, richiede solo un'indicazione. Il rema è una struttura imposta al reale, l'onoma è un'indicazione, un riferimento al reale, ossia una nota del reale.

Ma proprio la notazionalità è anche il limite ultimo della ricerca pizzutiana, forse per l'autore un segno del fallimento di questa ricerca: bisogna usare una moltitudine di nomi per indicare una parte del reale, bisogna quindi accostare i nomi, la natura sintattica —e sintassi vuole appunto dire disposizione- del linguaggio non è eludibile, inoltre per quanto possano essere variati, elaborati, forzati, la varietà degli accostamenti è limitata dalla necessità di rendere perspicua la notazione, la stessa natura referenziale del nome richiede una «deissi» appropriata.

Dunque non si può eliminare la necessità sintattica, se non altro perché mettere una pietruzza accanto all'altra implica di sapere quale pietra preceda l'altra: si può spingere la propria sperimentazione alla dissoluzione di quella sintassi di secondo grado che è rappresentata dalla struttura rematica del verbo, ma la sintassi basica, ossia l'accostamento di un nome all'altro, e l'inevitabile conseguenza che non tutto è accostabile a tutto, che l' "accostare» è un atto sottoposto a regole che permettono solo alcune descrizioni, non altre, è necessaria. Pizzuto giunge al limite estremo del linguaggio, dove la domanda linguistica diventa l'ostensione della necessaria sintatticità del linguaggio stesso, ossia la dimostrazione della nostra condanna alla parola.

\section{Un esempio da Ultime e Penultime}

La scelta di Ultime e penultime come testo saggio non è casuale, diversi sono i fattori che ci hanno persuasi a rivolgerci a questo testo: il testo, apparso postumo nel 1978 per i tipi del Saggiatore, già dalla prima ${ }^{40}$ edizione presenta alcuni tratti rilevanti, come la postfazione di Gianfranco Contini ${ }^{41}$ e la traduzione francese delle sole Ultime, a cura di Madeleine Santschi, che ne consentono un'indagine più calibrata rispetto al quasi coevo Giunte e virgole. Si consideri inoltre che la situazione dei testimoni manoscritti, indagata nell' introduzione all'edizione critica curata da Gualberto Alvino (2001), permette una più sicura restituzione dell'originale.

A questo si aggiunga almeno un altro elemento importante: la presenza di note autoriali sia ad Ultime sia a Penultime. ${ }^{42}$

39. Cfr. ibid.: significativamente uno dei significati che la famiglia lessicale di onoma comporta è anche quello di «biasimare», «disprezzare» ecc. (connesso anche con l'ittita hanhaniya «disprezzare» e con l'irlandese on "colpa»), il che riporta ad un certo tono di biasimo, che si fa a volte cupo altre volte nostalgico, della prosa pizzutiana, che pare quasi essere, a tratti, un atto di contrizione per il primitivo ed inevitabile peccato dell'accadere.

40. Anche se non migliore (cfr. la prefazione di G. Alvino a Pizzuto, Ultime..., cit.).

41. Nota per l'ultimo Pizzuto, ora in postfazione a Pizzuto, Ultime..., cit.

42. Cfr. Felicita Audisio "Le "Note" di Pizzuto a "Penultime" e una Tavola delle correzioni del testo", in Autografo, n. VII / 21 Nuova Serie, 1990, p. 85-108. 
In sintesi: la maggior fortuna critica e la migliore situazione filologica suggeriscono, in uno studio come il nostro, di rivolgersi ad Ultime e Penultime come esempio della prosa tardo-pizzutiana.

Discuteremo un unico testo - la prima «lassa» di Ultime: Dall'ombratratto dall'ottima edizione critica, apparsa per i tipi di Cronopio nel 2001, curata da Gualberto Alvino, ${ }^{43}$ che permette di ricostruire le diversi fasi di scrittura e costituzione del testo.

All'interno dell'opera la scelta è stata condotta applicando due parametri di massima: il primo potrebbe essere definito «parametro dell'irrilevanza linguistica»; questo parametro assume che nessuno dei passaggi di Ultime e Penultime sia linguisticamente più rilevante di un altro. Il secondo parametro potrebbe essere chiamato "parametro dell'interesse testuale»: i due passi stralciati presentano caratteristiche testuali che li rendono interessanti per la nostra ricerca.

La posizione incipitaria di Dall'ombra isola il testo sia rispetto ad Ultime sia rispetto a Penultime, tanto da poter concludere che il testo abbia un valore "programmatico» rispetto al progetto a dittico di Ultime e penultime; a suggerire tale conclusione è il richiamo gaddiano ${ }^{44}$ reso esplicito nella chiusura della "lassa». Si può così leggere Dall'ombra come un'implicita dichiarazione di poetica intesa a marcare la differenza fra poetica pizzutiana e poetica gaddiana ed allo stesso tempo capace di fornire al lettore la «chiave» della reale cifra stilistica dell'opera. ${ }^{45}$ Dall'ombra è dunque l'immagine «linguistica» della riflessione poetica e «metalinguistica» di Pizzuto, il che ne fa per noi un oggetto interessante.

Dall'ombra offre inoltre il vantaggio di squadernare dinnanzi al lettore un ampio ventaglio dei mezzi linguistici messi in uso da Pizzuto nella sua personalissima ricerca di un nuovo linguaggio sintattico, perciò riteniamo che il fatto di discutere da vicino questo solo passo del dittico composto da Ultime e penultime non sia un limite, vuoi per il valore di incipit assoluto che la pagina ha rispetto a tutta l'opera, come dicevamo sopra, vuoi per il valore esemplare della pagina da un punto di vista linguistico.

43. Gualberto Alvino è anche l'editore di Giunte e virgole (Antonio Pizzuto, Giunte e virgole, edizione critica di Gualberto Alvino, Roma: Fondazione Piazzola, 1996) e di Spegnere le caldaie (Antonio Przzuto, Spegnere le caldaie, edizione critica di Gualberto Alvino, Roma: Casta Diva, 1999).

44. Cfr. Peretti / Pizzuto, Pizzuto parla di Pizzuto, cit., p. 107-108.

45. Altro indizio del fatto che il testo possa essere visto sotto questa luce è, a nostro avviso, il confronto con i due testi che hanno esplicito valore di poetica: Sintassi nominale e pagelle e Paragrafi sul raccontare. In entrambi i casi la dichiarazione esplicita di un programma poetico è relegata in limine (o in exitu, nel caso dei Paragrafi) ad esempi di prosa ispirata dai principi esposti, addirittura, nel caso dei Paragrafi, l'"esempio» è un intero "romanzo" (Paginette). Non è quindi illecito proporre che, sotto il segno dell'estrema distillazione dei mezzi espressivi operata dall'ultimo Pizzuto, la dichiarazione di poetica sia sussunta ed «indicata» direttamente dal «farsi» del lavoro di scrittura. 


\subsection{Dall'ombra}

Prima, immediata notazione leggendo questo passo è il titolo in enjambemant, come Pizzuto in una notazione al testo segnala; ${ }^{46}$ seconda annotazione: il periodo che inizia con l'enjambement post titolo non ha verbo. ${ }^{47}$ Queste due primissime notazioni suggeriscono una riflessione sul fatto che Contini abbia battezzato «lasse» le sciolte pagine pizzutiane da cui sono composti i lavori che vanno da Pagelle in avanti: ${ }^{48}$ la lassa è lo schema poetico-narrativo adottato dalla primitiva poesia romanza, vuoi la Chanson de Roland in langue d'oül, vuoi il Poema de mio Cid, primissimo esempio letterario del castigliano, vuoi infine alcuni ritmi, ad es. quello Laurenziano, che stanno alle origini della letteratura in volgare italiano.

Costitutivi della lassa sono, dal punto di vista formale, la libertà metrica, poiché la lassa è di lunghezza variabile, per solito è priva di schema metrico, può essere basata tanto su assonanza (lassa assonanzata) quanto su rima (lassa rimata), oppure può usare e l'una e l'altra, ${ }^{49}$ e dal punto di vista contenutistico la non liricità del tema: la lassa è riservata alla narrazione epica, come nel Cid o nella Chanson, oppure, è il caso dei Ritmi antico italiani, si tratta di testi con contenuto laudativo o moraleggiante.

L'analogia consente di estendere a Pizzuto alcune delle caratteristiche proprie della lassa: l'antiliricità assoluta del contenuto anzitutto è un saldo analogon, così come base altrettanto salda dell'analogia pare la libertà formale. Da queste basi il processo analogico a partire da cui diciamo, sulla scorta del Contini, «lasse» quelle del Pizzuto, si estende alla struttura "assonanzata» dell'autore moderno. È questo il punto vitale: come la lassa si basava sul tenue tessuto dell'assonanza fonica che permetteva di costruire, nelle fasi antiche delle lingue romanze, la struttura, ancora incerta, del verso poetico, così, nella prosa pizzutiana, l' "assonanza» sintattico-semantica che governa l'assemblaggio delle parole sottratte alla struttura rematica dell'enunciato permette la costruzione di una nuova forma di espressione letteraria, che si situa volutamente oltre la cesura fra prosa e poesia. Mentre però la lassa medioevale come portato finale del passaggio, non solo linguistico, ma anche più generalmente culturale dal latino alle lingue volgari, tende ad un ordine di regolarità linguistica, la «lassa» pizzutiana, come risultato dello scompaginamento sintattico provocato dallo stesso Pizzuto, appare piuttosto come il residuo di un ordine linguistico costantemente messo in crisi dalla penna dell'autore ma che costantemente si impone a tale critica.

Consideriamo l'enjabement che crea la sequenza: «dall'ombra / ecco...»; sebbene il risultato più evidente sia l'isolamento in posizione di titolo del sintagma preposizionale, l'aspetto di maggior importanza è la posizione di preminenza isolata dell'avverbio «ecco» in chiara funzione presentativa.

46. Ricavabile dall'apparato critico dell'edizione Alvino.

47. Affermazione che verrà meglio circostanziata di seguito.

48. Cfr. Contini, Varianti..., cit., p. 621-625e ID., in Pizzuto, Ultime..., p. 271-282, inoltre il carteggio Pizzuto / Contini, Coup..., cit.

49. Esemplare in questo senso è proprio il Cid. 
Comunque Pizzuto avesse organizzato questo primo «periodo» di Dall'ombra, non avrebbe potuto in alcun caso esimersi dal compiere un atto indicale, linguisticamente rintracciabile, in grado di permettere al lettore di avere una leggibilità del passo; come infatti vedremo, la preminenza di «ecco» è vitale non solo per il primo "periodo", ma per tutta la prima pericope del brano che vede il gatto e la sua morte come tema della "narrazione» (all'incirca fino alla riga 9, quando inizia il passaggio che, in diverse fasi, porterà al finale «omaggio» gaddiano).

La posizione incipitaria in isolamento di "ecco» è dunque obbligata nel senso che abbiamo detto poc'anzi; a dimostrarlo c'è anche la traccia del processo di rastremazione ricostruibile a partire dalle varianti. ${ }^{50} \mathrm{Il}$ testo, prima di assumere definitivamente la forma che leggiamo, passa attraverso le varianti: "farsi avanti» e "or ecco farsi avanti», che, come è desumibile anche dalla copia fotostatica dell'incipit di Ultime, riprodotta a p. 21 dell'edizione Alvino, rappresentano le fasi precedenti del testo. Ė interessante il processo di composizione pizzutiano: non si tratta solo di asciugamento; l'apparato svela di più del semplice passaggio della lima, permette di osservare come l'autore ottenga di disinnescare e quindi distruggere la struttura del rema.

Il punto di partenza è la sequenza: «dall'ombra (/) farsi avanti», ${ }^{51}$ della cui normatività e direi «classicità» non si può dubitare. Pizzuto opera sul comune enunciato: «dall'ombra fasi avanti x», che è "classico» nella misura in cui rappresenta una sequenza normalmente accolta in qualunque forma tradizionale del

50. Desumibili sempre dall'utilissimo apparato dell'edizione Alvino.

51. Purtroppo non è possibile stabilire al di là di ogni dubbio se la notazione autoriale: «come titolo, con enjambement» riferita al sintagma: «dall'ombra» risalga effettivamente alla seduta di lavoro indicata con la data 23/IV/1973, oppure sia successiva, presumibilmente non oltre il 24/IV/1973, sessione nella quale Pizzuto, solitamente molto preciso nell'indicare ora e data di inizio e fine del lavoro di scrittura, definisce la sequenza finale: «dall'ombra / ecco innanzi». Sebbene l'apparato di Alvino avvalli l'ipotesi che la notazione risalga alla sessione del 23, il confronto con la riproduzione fotostatica dell'originale non fornisce una prova di avvallo definitivo all'interpretazione accolta in apparato. Per noi il particolare non è decisivo, tuttavia non è nemmeno irrilevante: se infatti la sequenza di partenza fosse effettivamente: «dall'ombra / farsi avanti» (ipotesi del 23), allora si dovrebbe ammettere che Pizzuto abbia in mente la struttura con enjambement fin da subito, il che implicherebbe, seguendo la ricostruzione delle fasi creative che delineiamo nel corso dell'articolo, che la posizione incipitaria post enjambement di «ecco» nel testo di arrivo fosse l'unica possibile. Se invece la sequenza di partenza fosse da interpretarsi come: «dall'ombra farsi avanti», senza enjambement (ipotesi del 24), allora si dovrebbe concludere che Pizzuto abbia avuto almeno tre possibilità compositive: accogliere nel testo definitivo la sequenza priva di enjambement, accogliere nel testo una versione in cui il sintagma preposizionale risulta isolato in posizione di titolo - versione effettivamente accolta nel testo di arrivo-, accogliere una versione capovolta dell'enjambement. Il fatto che si dia questa terza possibilità presuppone a sua volta che l'ipotesi dell'enjambement sia stata presa in considerazione solo alla fine del processo di disinnesco e distruzione della struttura rematica. Poiché, come diremo, la posizione post enjambement dell'avverbio implica il massimo abbassamento possibile della struttura rematica, sarebbe interessante decidere se tale risultato sia, per così dire, "programmato" già all'inizio del processo di scrittura, quindi con un restringimento a priori dell'ambito di lavoro linguistico (ipotesi del 23), oppure se l'ambito di lavoro linguistico sia il meno programmato possibile in vista di un risultato ottenuto per successive «divinazioni» (ipotesi del 24). 
«raccontare», nel senso della massima analiticità, esplicitando tutte le strutture temporali, spaziali, deittiche del rema, ottiene in questo modo la sequenza: «or ecco farsi avanti». Giacché tutte le implicazioni riguardo alla struttura spazio temporale sono expressis verbis in superficie, la nuova sequenza disinnesca la struttura rematica presentandola nell'interezza delle sue conseguenze spaziotemporali, e consentendo a Pizzuto di adoperarsi per evitare tali conseguenze.

Il processo di riduzione rematica procede lungo due direttrici: eliminazione delle informazioni temporali e spaziali, con passaggio da «avanti» ad »innanzi», quindi con sussunzione dei due valori, quello di spazio e di tempo, in un unico avverbio il cui valore ambiguo è amplificato dall'abbassamento massimo possibile del tratto esistenziale del rema; proprio questo indebolimento della predicazione esistenziale è la seconda direttrice su cui si muove Pizzuto. Se ci soffermiamo un poco su questa seconda mossa della strategia pizzutiana, possiamo agevolmente comprendere quale sia la soluzione del complesso rapporto che la prosa pizzutiana intrattiene col rema e con le forme verbali.

A monte c'è l'esclusione delle strutture di tempo, aspetto e modalità, il che porta all'eliminazione delle forme finite del verbo, più a valle però troviamo anche il tentativo di eliminare la struttura agentiva del predicato, mettendo così in crisi definitiva anche quella distinzione fra agente e paziente che è definita dall'uso della forma verbale. Tuttavia, l'eliminazione della distinzione fra agente e paziente non è così agevole come l'elisione delle strutture di tempo, aspetto e modo, poiché quest'ultima struttura verbale riguarda la predicazione esistenziale del verbo. Quindi, laddove non sia possibile altro mezzo per istituire l'esisten$\mathrm{za}$, si ricorre all'uso debole del verbo per mezzo di infinito e participio, laddove invece sia possibile una forma assolutamente nominalizzata di enunciato, si elide senz'altro il verbo, ricorrendo ad un segnale testuale ${ }^{52}$ che stabilisce l'esistenza di qualcosa: nel nostro caso si tratta dell'avverbio «ecco», usato in funzione di puro deittico; l'elemento indicale «ecco» assume così valore puramente presentativo e quindi sostitutivo del predicato nella predicazione di esistenza.

Siamo così venuti a toccare il limite di tensione estremo della dialettica che intercorre fra la struttura interna del linguaggio e la poetica guastelliana e fenomenista di Pizzuto: tale limite di attrito è la necessità della predicazione esistenziale, essenziale alla poetica pizzutiana, ma organizzata dal linguaggio umano secondo una disposizione sintattica di elementi linguistici che devono essere connessi in un modo dato per significare tale esistenza.

Grazie alla rigorosa definizione del concetto di «narrare» come registrazione dell'evento libera da ogni valutazione e, verrebbe da dire, da ogni «ideologia", Pizzuto ottiene una riduzione della semantica alla sola predicazione di esistenza intesa come istituzione esistenziale dell'evento tracciato nella "narrazione», tale narrazione lo lega indissolubilmente ad una sintassi che è sintassi dell'esistenziale: come dimostra il passo che stiamo analizzando, una volta

52. Adotto questa definizione perché ritengo che metta meglio in evidenza l'uso di squisita coesione in termini contiani (cfr. Conte, Condizioni..., cit.), si potrebbe anche dire con nettezza, in termini petofiani (cfr. Petőfi, Scrittura..., cit.) testuale che l'autore fa dei segnali discorsivi. 
trovato il modo di definire l'esserci di qualcosa, la disposizione enunciativa si dispone attorno al nucleo forte della predicazione esistenziale. Nella frase pizzutiana vi è innanzi tutto la posizione della predicazione, poi, attorno a questo nucleo, che può essere rappresentato da un semplice avverbio, avviene, secondo strategie di rilevanza che potremmo definire, in termini aristotelici, accidentali, il disporsi a corona dei nomi.

In questa lotta semantico / sintattica però Pizzuto è limitato dalle necessità del mezzo linguistico, che richiede talora espressione di concetti di tempo, agente, modo, aspetto non sempre elidibili.

Uno degli aspetti non elidibili è quello che potremmo chiamare tempo interno dell'azione: ${ }^{53}$ si consideri l'inizio del secondo periodo di Dall'ombra: «Raggiunta l'opposta muraglia, rimanervi stecchito ritto...». Il periodo prende l'avvio da un participio passato - «raggiunta»— che segna una sequenza del tipo prima / dopo, in cui l'evento del rimanere stecchito segue quello, descritto nel precedente periodo, dell'attraversare la strada, di cui il participio «raggiunta» segna il culmine.

$\grave{E}$ interessante analizzare sia la strategia nominale che descrive l'evento dell'attraversare la strada, sia il costituirsi di questa sorta di consecutio temporum che suddivide in diverse pericopi serialmente organizzate il macro-evento che porta il titolo Dall'ombra. Il testo è il seguente:

Dall'ombra / ecco innanzi, tesi fiduciale prevalsa (participio con esclusivo valore aggettivale), austero soriano ove guadosa carreggiata, siccome carico di bagagli, greve la barda, ${ }^{54}$ a presidio ovvia subita diffidenza; and eat no fish.

53. Molti sono gli approcci narratologici al tempo, su tutti quello di Weinrich (Harald WeINRICH, Tempus. Besprochene und erzählte Welt, Stuttgart: Kolhammer, 1964), nella nostra brevissima analisi del tempo in Pizzuto però a far da sfondo è un approccio latamente formalizzato al tempo, come quello che è possibile ricavare da Andrea Bonomi / Sandro Zucchi, Tempo e linguaggio. Introduzione alla semantica del tempo e dell'aspetto verbale, Milano: Bruno Mondadori, 2001. Per una guida generale riguardo i diversi approcci al tempo si guardi l'utile, anche se disordinato, Robert Binnick Time and the Verb: a Guide to Tense and Aspect, New York / Oxford: Oxford University Press, 1991.

54. En passant facciamo notare l'anfibologia della sequenza: "greve la barda». Il senso è che la «barda» degli immaginati bagagli che l'andatura guardinga del gatto induce a pensare gravino la soma dell'animale («siccome carico di bagagli») sia "greve», quindi "greve» è aggettivo e cultismo per pesante, e «barda» è nome e metafora colta per "fardello» («barda» = armatura completa del cavallo, da qui il processo metaforico per cui il carico dei bagagli schiaccia il gatto così come la pesante armatura rischiava di far stramazzare il cavallo nel corso delle battaglie o delle giostre medioevali; non si dimentichi che il gatto, alla fine della traversata, muore, appunto stramazzando contro un muro e ricomponendosi a mo' di bassorilievo mesopotamico). È però a prima vista possibile una seconda interpretazione: «barda» sarebbe la terza persona indicativa presente di «bardare», "greve» sarebbe uso avverbiale dell'aggettivo, che sostituirebbe, come possibile, un possibile «grevemente», «la» a questo punto sarebbe un pronome dimostrativo clitico anaforico di "carreggiata». Da questa seconda ipotesi si cava il seguente senso generale: un gatto, là dove la carreggiata permette l'attraversamento ("guadosa»), come se fosse carico di bagagli, grevemente la (quella, ossia la carreggiata) attraversa come se fosse un ornamento troppo vistoso per quella (ancora la carreggiata). La seconda interpretazione è da scartare, non solo perché l'interpretazione bardare / attraversare troppo 
Raggiunta l'opposita muraglia, rimanervi stecchito ritto, quasi fittile mesopotamica sagoma.

Dopo le prime quattro parole di inizio, già ampiamente discusse, troviamo il soggetto dell'asserzione esistenziale implicita in «ecco»: si tratta di un gatto, paragonato al protendersi in avanti dello slancio di fiducia. L'inversione del gruppo che ha funzione generalmente attributiva («tesi fiduciale prevalsa») rispetto al soggetto dell'esistenziale, il «soriano» accidentalmente «austero», indica che la qualità espressa dal gruppo attributivo rappresenta l'intima caratterizzazione "ontologica» del nostro soggetto, che dunque si trova tutto proteso in avanti («ecco innanzi»), in accordo col suo élan di fiducia ormai pienamente accolta ("prevalsa»), laddove è possibile attraversare una strada (ossia dove la "carreggiata» si fa "guadosa»). A questo punto il protendersi avanti del nostro soggetto si blocca: schiacciato dalla "greve barda» dei bagagli, l'essere slanciato «innanzi» del nostro gatto si blocca, rallenta per un' "ovvia» diffidenza. È ambigua, magistralmente ambigua, questa diffidenza posta «a presidio» non si sa se dell'attraversamento o del fardello che grava l'animale, e «subita», perché improvvisa, subitanea appunto, ma anche indotta, ingenerata sebbene non voluta, ossia «subita». Chiude il periodo, con oracolarità ancor più marcata, una citazione da King Lear: ${ }^{55}$ allo stesso tempo fatto, constatazione -il gatto non mangia più pesce (ossia è morto) — e professione.

Ci sono tutti gli atomi dell'evento, che è «narrato», ossia ripreso e tratteggiato in ogni suo elemento essenziale: il soggetto (gatto), l'attraversamento, la morte, indicata come atomo dell'evento «un gatto dopo aver attraversato la strada muore» per mezzo di una citazione, altro grimaldello usato da Pizzuto per evitare la struttura rematica, ${ }^{56}$ ma manca la chiave di lettura. La chiave di lettura è fornita anzitutto dal participio «raggiunta» con cui si inizia il secondo periodo: la funzione tempo / aspettuale del participio - il passato ci indica che l'azione precedente si è conclusa e l'Aktionsart del verbo «raggiungere» vuole che vi sia una qualche azione di movimento di cui il raggiungimento sia il fine- ci permette di reinterpretare, nel senso di Conte, ${ }^{57}$ la «narrazione» precedente come narrazione di un attraversamento.

Pizzuto non poteva qui elidere la struttura interna del tempo, pena l'insignificanza, l'esigenza sintattica gli impone di esplicitare quale sia la connessio-

vistosamente sembra troppo forzosa in questo contesto, soprattutto se confrontata all'interpretazione barda / bagaglio pesante come un'armatura, che peraltro, come notavamo, in linea con il prosieguo della «narrazione», ma anche perché non è necessaria al testo. Il bisticcio, indotto dall'assenza di una struttura rematica ed artatamente provocato da Pizzuto tramite il ricorso ad una ripetuta discrepanza fra lectio difficilior («barda» nome in assenza di verbo e peraltro arcaismo) e lectio facilior («barda» verbo che apparentemente soddisfa l'esigenza di perspicuità del lettore e riflette l'uso comune della lingua) è l'ennesima, ironica dissoluzione della struttura rematica, di cui qui l'autore mostra l'inanità.

55. È Pizzuto stesso ad indicarla in nota, con le parole: «...Atto I, scena IV, Kent «Faccio professione ... di non mangiare pesce.....: si tratta però di ricostruzione.

56. Alla citazione in questo senso dedicheremo però altre pagine in altra sede.

57. Cfr. Conte, Condizioni..., cit. 
ne fra lo stato in luogo del gatto («ove guadosa carreggiata») e i vari «attributi» che lo descrivono ("....siccome carico di bagagli, greve la barda, a presidio ovvia subita diffidenza...») e quale la connessione interna dei vari attributi l'uno con l'altro. Il processo di reinterpretazione così attivato è dunque complesso ma sicuro: complesso, perché si tratta di ricostruire la struttura enunciativa in assenza di rema, sicuro perché l'autore ha lasciato tutti e solo quei segnavia che ci guidano nella ricostruzione univoca dell'evento. In questa maniera si ottiene quella distruzione del rema come giudizio premesso all'evento pur salvando il rema stesso come struttura dell'evento.

Lievemente, ma significativamente diverso il processo reinterpretativo implicato dell'infinito «rimanervi stecchito». La citazione conclusiva del precedente periodo rimaneva come pendente, non essendo chiaro quale rilevanza avesse il non mangiare più pesce: lo sappiamo ora, opponendo a quella citazione la semplice notazione: «....rimanervi stecchito ritto...»; la chiave di lettura è data non per mezzo di ricostruzione della struttura interna dell'evento, ma per contrapposizione di due informazioni attinenti all'evento, di due situazioni che non si potrebbero dare se non si fosse verificato precisamente quell'evento che non è predicato, in questo caso il morire del gatto. Qui a fungere da segnavia non sono gli elementi residuali dell'evento inclusi nella narrazione, ma è la contrapposizione fra parti del testo complessivo, ossia la macro-struttura della pagina.

A questo punto può seguire una lunga catena di periodi non rematici, su cui non ci soffermiamo, che "narrano» la natura dell'evento morte. Alla riga 9 si assiste ad un passaggio tematico, dalla morte verso la vita, il passaggio a questa nuova parte del testo, che può anche essere vista come una sorta di dichiarazione / esposizione / premessa dell'intento poetico di Ultime e penultime, è marcato da un infinito:

Interrogare dalla porta novella età addentratesi, onde non un attimo, pur il contiguo, prendibile; propiziarla rimorsi per tenuissime colpe, l'illusione complementare, picasse pennellate aspre vivide ristorative, con dispregio di quanto offertoci, lodi al vendere, superstizioni augurali. Meglio sicché ogni ultimo del suo penultimo, non aversi regimi oltre industre presagio.

Molte sono le differenze fra il ductus di questa pericope e quello della precedente, tutta giocata sull'assenza di verbi:

Raggiunta l'opposita muraglia, rimanervi stecchito ritto, quasi fittile mesopotamica sagoma. Né da novero. Altri cataloghi; poi gli incommensurabili. Squallida corporeità suddita a infiniti processi tosto operanti, di stanziale favilla singoli nati: per ciascuno sua propria araldica, in aneddoti raccolti entro favoleggiato catasto. E sotto quel disagio precorritrice tristezza. ${ }^{58}$

58. Esclusa la ripetizione della finale della prima pericope del testo ( Raggiunta l'opposita muraglia, rimanervi stecchito ritto, quasi fittile mesopotamica sagoma.»), si noti come l'unica struttura debolmente verbale sia rappresentata dalla sequenza «infiniti processi tosto operanti», con participio congiunto che ha però più valore aggettivale che verbale. Ancora una volta, proprio all'inizio di questa pericope, Pizzuto ironeggia sul rema nella sequenza 
Notiamo immediatamente un fatto: arematica assolutamente è la pericope dedicata alla morte, non quella in cui si affrontano i temi del vivere. Fatto questo poetico ma anche «linguistico» e simultaneamente fenomenico: la presenza di eventi (vita) ha una sua struttura, che, pur non essendo temporalmente descrivibile ("onde non un attimo, pur il contiguo, prendibile»), è tuttavia legata ad una struttura, foss'anche quella delle "picasse pennellate», che, come spiega Pizzuto in nota: «tracciando un occhio qui e un altro là, rendono tutto più vivo». Questa struttura Pizzuto la cerca attraverso quello smontaggio del rema linguistico, con le sue strutture temporali, agentive e modali, a favore di un rema che si potrebbe definire puramente eventuale / narrativo che abbiamo cercato di descrivere sopra.

Ė come se Pizzuto tracciasse un'opposizione, complementare e contigua a quella fra linguaggio e metalinguaggio, fra eventualità e metaeventualità: dalla parte della metaeventualità, che implica un giudizio, in senso gnoseologico e kantiano, sull'evento vi è in realtà il linguaggio, che non è in grado di esprimere l'eventualità pura perché parla dell'evento mediante le strutture sintattico / gnoseologiche che ne costituiscono le regole; a questa mediatezza eventuale del linguaggio fa da contraltare l'immediatezza eventuale del metalinguaggio, che mettendo in chiaro le regole del gioco linguistico ne svela la contraffazione eventuale, permettendo così di creare un'espressione linguistica dell'evento in accordo con l'evento stesso, ossia un «linguaggio» dell'essere. Non è azzardato assumere che la prosa di Pizzuto sia una sorta di filosofia del linguaggio applicata; non una teoretica, ma una pratica dell'andare verso il «linguaggio» come capacità linguistica dell'essere.

Lungo questo cammino, che travolge ogni profferta del mondo linguistico («con dispregio di quanto offertoci, lodi al vendere, superstizioni augurali»), unico criterio è il farsi sempre più estremo del cammino, l'opera di discesa $\mathrm{e}$ attacco ai fondamenti linguistici della contraffazione ontologica infatti è inarrestabile: «meglio sicché ogni ultimo del suo penultimo», considerazione questa che ci permette di interpretare il titolo, e con esso l'opera: ogni pagina scritta è ultima, rispetto alla penultima, ma anche penultima di una nuova ultima che nascerà da una nuova discesa, dunque Ultime e penultime, con ironica inversione dell'ordine di sequenza temporale della composizione, e non mai «penultime» e «ultime», perché pagine «ultime» non ve ne sono, vi sono solo le ultime "penultime» in ordine di tempo, ma un tempo che per Pizzuto non ha importanza.

Su questa strada infinita c'è un precedente, qualcuno cui rivolgere un omaggio, seppure salace, seppure ambiguo, qualcuno che può consigliare, forse solo a metà, qualcuno che rappresenta una soglia di partenza, Carlo Emilio Gadda:

«né da novero» nello stesso modo in cui aveva fatto nella sequenza "greve la barda» (cfr. nota precedente). Qui, a differenza che nella sequenza precedente, la lettura verbale è ancora più improbabile, ma l'anfibologia serve all'autore in maniera più strumentale per sottolineare il valore di perifrastica passiva della sequenza: la morte infatti non è cosa da annoverarsi nel catalogo della vita affrontato in Ultime e penultime, riguarda altro, i foscoliani incommensurabili. 
Tu riconsigliarmi dalla statura, o bocca bocca bella con i baffini furieri, imo sguardo quanto la Fossa, fulmineo l'inestimabile sorriso. Hélas. Lo sfincterallasvega. Ingravallo. Ed empiti di tematiche wagneriane

Spiega Pizzuto stesso l'ironia dell'omaggio, in cui la «statura» del Gadda subito s'abbassa a un che da operetta («o bocca bocca bella...») condita dal grottesco dei baffetti hitleriani, ${ }^{59}$ come specifica Pizzuto in nota, si condensa tutta nella creazione del neologismo "sfincterallasvega» (sfintere le cui funzioni portano ad una somiglianza con le slot-machines dei casinò di Las Vegas), per cui rimandiamo a Pizzuto stesso. ${ }^{60}$

C'è però l'altra faccia dell'ironia, celata dal riserbo reticente dell'autore, c'è l'opus continuum del Pasticciaccio, c'è la mescidazione assoluta dei linguaggi e dei registri (gli «empiti di tematiche wagneriane», riguardo ai quali Pizzuto, ancora una volta in nota, commenta: «in Gadda i dialetti si mescolano come i temi wagneriani che annunciano i vari eroi»), c’è il sorriso e lo sguardo di Gadda, c'è insomma quell'opera eversiva di interrogazione assoluta, radicale che Gadda propone al livello di quella che abbiamo definito «retorica» e Pizzuto riconosce e trasborda nel linguaggio.

\section{Conclusioni}

Dove ci ha portato questa breve cavalcata nella sintassi dell'ultimo Pizzuto? Non certo ad una conclusione, piuttosto ad un inizio.

Abbiamo visto che, al di là della questione delle grandi narrazioni, la domanda al linguaggio può essere praticata, oltre che posta, direttamente al linguaggio stesso, perciò non alla sua struttura ideologica ma alla struttura gnoseologica: abbiamo visto che tale pratica del domandare linguistico va incontro al limite ultimo della regola che non ammette altra eversione, pena il venire meno del linguaggio stesso, come avviene nella patologia psicotica.

Ma ora che alcuni punti li abbiamo fissati, qual è il sedimento che l'opus infinitum e penultimo, ma non fallimentare, di Pizzuto lascia? È questa la domanda finale a cui siamo arrivati, da cui partire verso nuove ricerche. ${ }^{61}$

59. Pizzuto ha certamente in mente una fotografia della metà anni venti in cui appare un Gadda ancora giovane, con corti baffetti quadrati, che avrebbe poi tagliato, vestito con britannica e composta eleganza.

60. Cfr. Peretti / Pizzuto Pizzuto parla di Pizzuto, cit., p. 107-108.

61. Ricerche affrontate nel nostro: Extragrammaticalità. Note linguistiche, critiche e filosofiche sull'ultimo Pizzuto, Torino: Ananke, 2012. 


\section{Bibliografia delle opere di Antonio Pizzuto}

\section{Romanzi}

Ultime e penultime, edizione critica di Gualberto Alvino, Napoli: Cronopio, 2001. Pagelle I, Milano: Il Saggiatore, 1973.

Pagelle II, Milano: Il Saggiatore, 1975.

Giunte e virgole, edizione critica di Gualberto Alvino, Roma: Fondazione Piazzola, 1996.

Spegnere le caldaie, edizione critica di Gualberto Alvino, Roma: Casta Diva, 1999.

Si riparano bambole, a cura di Gualberto Alvino, Palermo: Sellerio, 2001. Paginette, a cura di Antonio Pane, Firenze: Polistampa, 2002.

Ravenna, a cura di Antonio Pane, Firenze: Polistampa, 2002.

Sul ponte di Avignone, a cura di Antonio Pane, Firenze: Polistampa, 2004.

Signorina Rosina, a cura di Antoni Pane, Firenze: Polistampa, 2004.

Testamento, commento di Antonio Pane, Firenze: Polistampa, 2009.

Sinfonia (1927), a cura di Antonio Pane, S. Angelo in Formis: Laveri, 2009.

\section{Saggi}

«Paragrafi sul raccontare», in Id., Paginette, Firenze, Polistampa, 2002, p. 119-120. «Dello scrivere difficile», Nuovi Argomenti, n. 14, 1969, p. 55-64.

\section{Epistolari}

Pizzuto, A. / Contini, G., Coup de foudre. Lettere (1963-1976), a cura di G. Alvino, Firenze: Polistampa, 2000.

Pizzuto, A. / Nencioni, G., (1998) Caro Testatore, Carissimo Padrino. Lettere (1966-1976), a cura di G. Alvino, Firenze: Polistampa, 1998.

Pizzuto, A. / Scheiwiller, V., Le carte fatate. Carteggio (1969-1975), a cura di C. Gibellini, Libri Scheiwiller, 2005.

Pizzuto, A. I Spinelui, S., Ho scritto un libro ... Lettere (1929-1949), a cura di A. Pane, Palermo: Nuova Ipsa, 2001.

Pizzuto A. / Peretti P., Pizzuto parla di Pizzuto, con un'introduzione di W. Pedullà, Cosenza: Lerici, 1978. 